\title{
Laser cooling of solids: Towards biomedical applications
}

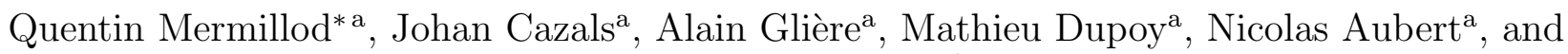 \\ Stephan Chabardès ${ }^{\mathrm{a}, \mathrm{b}}$ \\ ${ }^{a}$ Univ. Grenoble Alpes, CEA, LETI, F38000 Grenoble, France \\ bService de Neurochirurgie, CHU Grenoble Alpes, F38000 Grenoble, France
}

\begin{abstract}
Focal cooling is a promising alternative therapy for intractable focal epilepsies, avoiding the irreversible neuronal damages induced by resection surgery. However, due to thermal conduction losses, local cooling of a deep brain region remains a challenging objective for thermoelectric or fluidic technologies. Here, we investigated the viability of an optical micro-cooler based on anti-Stokes refrigeration of ytterbium doped YLF crystals, taking into account the medical constraints for implantable device. We realized significant cooling under atmospheric pressure and developed a solution drastically reducing the harmful fluorescence heating of brain-like liquids below $2 \mathrm{~K}$, thus demonstrating the relevance of this technology for biomedical applications.
\end{abstract}

Keywords: Laser cooling of solids, Optical refrigeration, Anti-Stokes fluorescence, Intractable focal epilepsies, Active implantable medical devices

\section{INTRODUCTION}

During the past decades, optical refrigeration based on anti-Stokes fluorescence of rare-earth doped glasses,${ }^{1-6}$ crystals $^{7-13}$ and semiconductors ${ }^{14}$ have shown very promising results at low temperature under vacuum conditions, and even sometimes under atmospheric pressure. ${ }^{15,16}$ Active researches have recently demonstrated an all-solid-state optical cryo-cooler, able to cool an infrared sensor at cryogenic temperatures without vibrations. ${ }^{17}$ This demonstration paves the way to spatial infrared imaging applications, where reliability and lifetime are crucial.

Apart from spatial applications, the compactness, vibration-free and focal cooling interests of optical microcoolers seem very suitable for active implantable medical devices (AIMD), especially in focal epileptic seizures treatment. ${ }^{18,19}$ In that case, optical cooling of a deep brain region without thermal conduction losses has tremendous benefits compared to thermoelectric or fluidic cooling.

Here, we investigated the viability of an optical micro-cooler based on ytterbium doped YLF crystals, taking into account the medical constraints for implantable device: biocompatibility, reduced size and negligible heating. To do so, we reproduced the biological environment, in order to evaluate the cooling efficiency of the crystals in real conditions, taking into account the harmful heating due to fluorescence absorption.

We firstly demonstrated significant cooling under atmospheric pressure, which represents a first step towards AIMD since the complex vacuum constraint is removed. Besides, we revealed a substantial fluorescence heating in different liquids reproducing human brain properties. To fix this drawback, we developed a solution to reduce drastically the amount of escaping fluorescence, thus limiting the liquids heating below $2 \mathrm{~K}$, and demonstrating the relevance of this technology for biomedical applications.

\section{FOCAL COOLING FOR EPILEPSY}

Epilepsy is a disabling and severe neurological disorder that affects about $1 \%$ of the world's population, making it one of the most common neurological diseases. ${ }^{20,21}$ For most patients, epilepsy can be adequately controlled with medication treatments. However, 30 to $40 \%$ of patients continue to experience disabling seizures or unacceptable drug effects. ${ }^{22,23}$ About $10-50 \%$ of these drug-resistant patients are candidates for resection surgery, ${ }^{24}$ with a rate of seizure freedom of $40-90 \%$, depending on underlying pathology. However, it remain millions of patients

* quentin.mermillod@gmail.com 
who cannot be treated by resective surgery or declare recurrent seizures despite surgery. Only a small part of them $(<10 \%)$ achieve seizure freedom with vagus nerve stimulation therapy after failed resection. ${ }^{25}$ For this uncovered and severely disabled population, there is a pressing need for alternative therapies.

Promising alternative therapies for medically refractory epilepsy are actually in progress. One of them consists in the neurostimulation of the anterior nucleus of the thalamus as shown in Fig. 1(a). A reduction in seizure frequency of more than $50 \%$ was demonstrate in almost $70 \%$ of implanted patients during 5 years of follow-up, and $16 \%$ of subjects were seizure-free for at least 6 months. ${ }^{26}$

An other emerging alternative therapy consists in the focal cooling of the epileptic foci during the seizure. Recent studies have demonstrated that focal cooling of the cortical surface of the brain to temperature slightly around $300 \mathrm{~K}$ terminates epileptic discharges without inducing irreversible neurophysiological dysfunctions or neuronal damage in the brain tissues. ${ }^{18,19,27}$ In these studies, Peltier-type thermoelectric devices were used to cool the cortical surface. However, this technology is not suitable for a fully implantable device to cool efficiently deep brain regions as it is necessary to evacuate the heat from the hot side.

The technological challenge is therefore to design a localized cooling system that can be implanted in the brain in the long-term, allowing rapid and effective inhibition of the epileptic foci and meeting all the AIMD constraints gathered in the ISO14708-1 standard and in particular: (i) tissue heating bellow to $2 \mathrm{~K}$, (ii) flexible probe with contacts of about $1.3 \mathrm{~mm}$ in diameter, (iii) biocompatibility. The energy consumption, compatible with the use of implantable batteries must also be taken into account.

With this in mind, the long-term objective is to develop a micro-cooler based on laser cooling technology, that will then be integrated into an AIMD prototype compatible with preclinical studies, as shown schematically in Fig. 1(b). The probe would integrate electrode arrays that can ultimately record neural activity, detect seizures and reduce the temperature of surrounding tissues. The main drawback of this technology is the escaping fluorescence of the crystal, which is absorbed by the tissues. Therefore, a careful study of the fluorescence absorption by the tissues must be carried out to limit harmful heating.
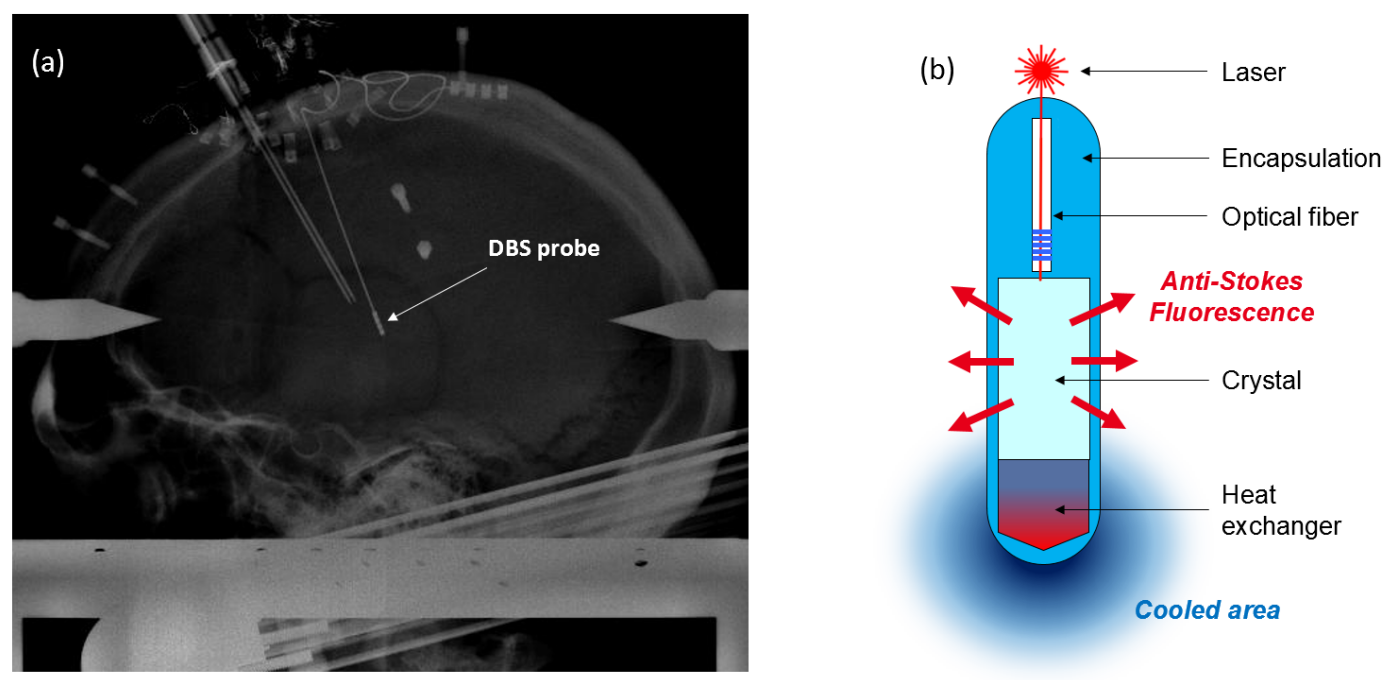

Figure 1. Alternative therapies for medically refractory epilepsy. (a) X-ray image of a surgical implantation performed at Clinatec (CEA Grenoble, France) of a deep brain stimulation (DBS) probe in the anterior nucleus of the thalamus. (b) Schematic illustration of the cooling probe based on optical refrigeration.

\section{THEORETICAL REVIEW}

The principle of cooling a solid through the anti-Stokes fluorescence was theoretically predicted by Peter Pringsheim in $1929 .{ }^{28}$ Fig. 2 schematically describes this process for $4 f$-electronic states of $\mathrm{Yb}^{3+}$ ions (see inset), with 
the associated absorption (red curves) and fluorescence (blue curve) spectra. The first step is the absorption of weakly entropic photons of energy $h \nu$ in resonance with the lowest-energy $\delta E_{g}$ between the fundamental and excited states manifolds. Then, the excited atom thermalizes very quickly ( $\sim$ ps) by absorbing phonons from the lattice. Finally, the atom returns to its fundamental state by radiative de-excitation $(\sim \mathrm{ms})$, producing an anti-Stokes fluorescence by spontaneous emission of average energy $h \tilde{\nu}_{f}$ higher than the energy of the absorbed photons. This excess energy, shaded in red, is removed from the solid through the fluorescence and causes a net cooling proportional to the amount of photons escaping from the solid.

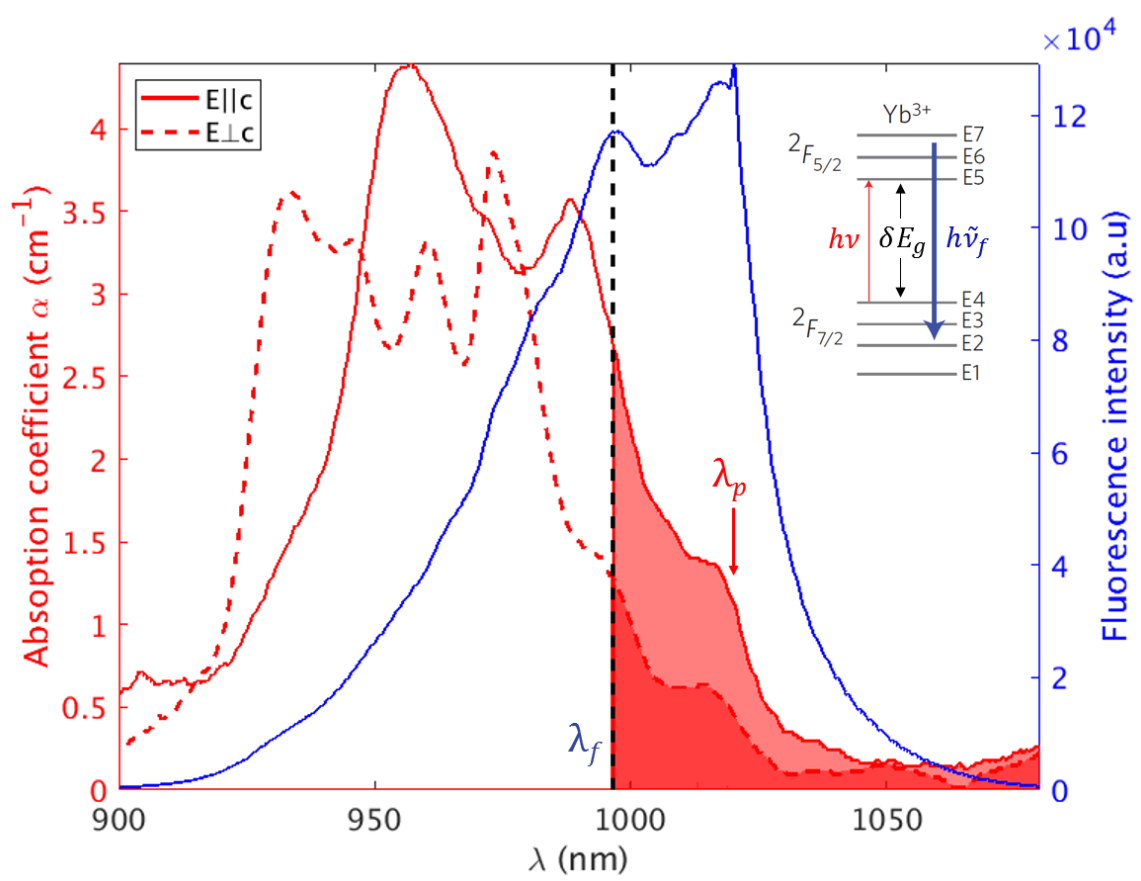

Figure 2. Room temperature absorption coefficient $\alpha=\alpha_{r}+\alpha_{b}$ (red) for parallel (solid) and perpendicular (dashed) polarizations and fluorescence emission spectra (blue) for a pump at $\lambda_{p}=1020 \mathrm{~nm}$ polarized along the $c$-axis. Cooling region where $h \nu<h \tilde{\nu}_{f}$ is shaded in red. Inset: energy levels of $\mathrm{Yb}^{3+}$ ions containing seven Stark manifolds (E1-E7) distributed over the ground-state ${ }^{2} F_{7 / 2}$ and the excited state ${ }^{2} F_{5 / 2}$.

For each photon absorbed, the fractional cooling energy or cooling efficiency is ${ }^{1}$

$$
\eta_{c}=\frac{h \tilde{\nu}_{f}-h \nu}{h \nu} \approx \frac{k_{B} T}{\delta E_{g}},
$$

where $k_{B}$ is the Boltzmann constant, $T$ is the temperature and $\delta E_{g}$ is the energy separation of the fundamental transition $\mathrm{E} 4 \rightarrow \mathrm{E} 5 . \delta E_{g} \approx 1.2 \mathrm{eV}$ for $\mathrm{Yb}^{3+}$ ions, resulting in a cooling efficiency of $\eta_{c} \sim 2 \%$ at room temperature, when pumping at resonance $\left(h \nu=\delta E_{g}\right)$. In this ideal case, the cooling efficiency increases linearly by decreasing the energy of pump photons. In reality, the cooling efficiency is limited by non-radiative recombination processes and background absorption from residual impurities that cause heating of the solid.

Three conditions are necessary to achieve significant cooling: (i) high external quantum efficiency to reduce nonradiative recombination processes, (ii) high resonant absorption, in order to absorb the largest amount of pump photons, (iii) high-purity host materials to minimize the residual absorption. Taking these three conditions into account, the real cooling efficiency can be rewrite as follows ${ }^{29}$

$$
\eta_{c}=\eta_{e x t} \eta_{a b s} \frac{h \tilde{\nu}_{f}}{h \nu}-1,
$$


where $\eta_{e x t}$ and $\eta_{a b s}$ are respectively the external quantum efficiency and the absorption efficiency defined as

$$
\begin{gathered}
\eta_{e x t}=\frac{\eta_{e} W_{r}}{\eta_{e} W_{r}+W_{n r}}, \\
\eta_{a b s}=\frac{\alpha_{r}}{\alpha_{r}+\alpha_{b}},
\end{gathered}
$$

where $\eta_{e}$ is the extraction efficiency, $W_{r}$ and $W_{n r}$ are respectively the radiative and non-radiative recombination rate, $\alpha_{r}$ and $\alpha_{b}$ are the resonant and background absorption respectively. Net cooling occurs when $\eta_{c}>0$, which leads, for a pump detuning of $h \tilde{\nu}_{f}-h \nu=k_{B} T$, to the following condition

$$
\eta_{e x t} \eta_{a b s}>1-\frac{k_{B} T}{h \nu}
$$

For $\mathrm{Yb}^{3+}$ ions, cooling occurs when $\eta_{\text {ext }} \eta_{a b s}>98 \%$ (99\%) at $300 \mathrm{~K}(100 \mathrm{~K})$. Theses requirements are possible in high purity host materials (i.e. $\alpha_{b} \rightarrow 0$ ) with low phonon energy compared to $\delta E_{g}$ to avoid multi-phonon relaxation (i.e. $W_{n r}<<W_{r}$ ). $\mathrm{Yb}^{3+}$ ions doped YLF crystals grown by Czochralski process meet these conditions and actually remain the most efficient cooling materials. To date, the lowest cooling temperature of $91 \mathrm{~K}$ was achieved with $10 \%$ wt. $\mathrm{Yb}^{3+}:$ YLF crystals under vacuum environment. ${ }^{12}$ The same kind of crystals provided by AC Materials (Tarpon Springs, United Sates) were used for this study.

Fig. 3(a) shows the temperature dependence of the fluorescence spectra and Fig. 3(b) the cooling efficiency of these crystals. At room temperature, the maximum cooling efficiency is obtained for wavelengths higher than the E4 $\rightarrow$ E5 transition, corresponding to the wavelength $\lambda=1020 \mathrm{~nm}$. Following the Boltzmann distribution $\propto\left[1+\exp \left(\delta E_{g} / k_{B} T\right)\right]^{-1}$, absorption efficiency decreases exponentially with temperature, which is directly visible in the fluorescence intensity. Consequently, the maximum cooling efficiency is blue-shifted to $1020 \mathrm{~nm}$ and gradually goes to zero until the global minimum achievable temperature (gMAT) is reached (not shown on Fig. 3(b)). Note that Stark manifolds appear more sharply as the temperature decreases, since the inhomogeneous broadening fades away.
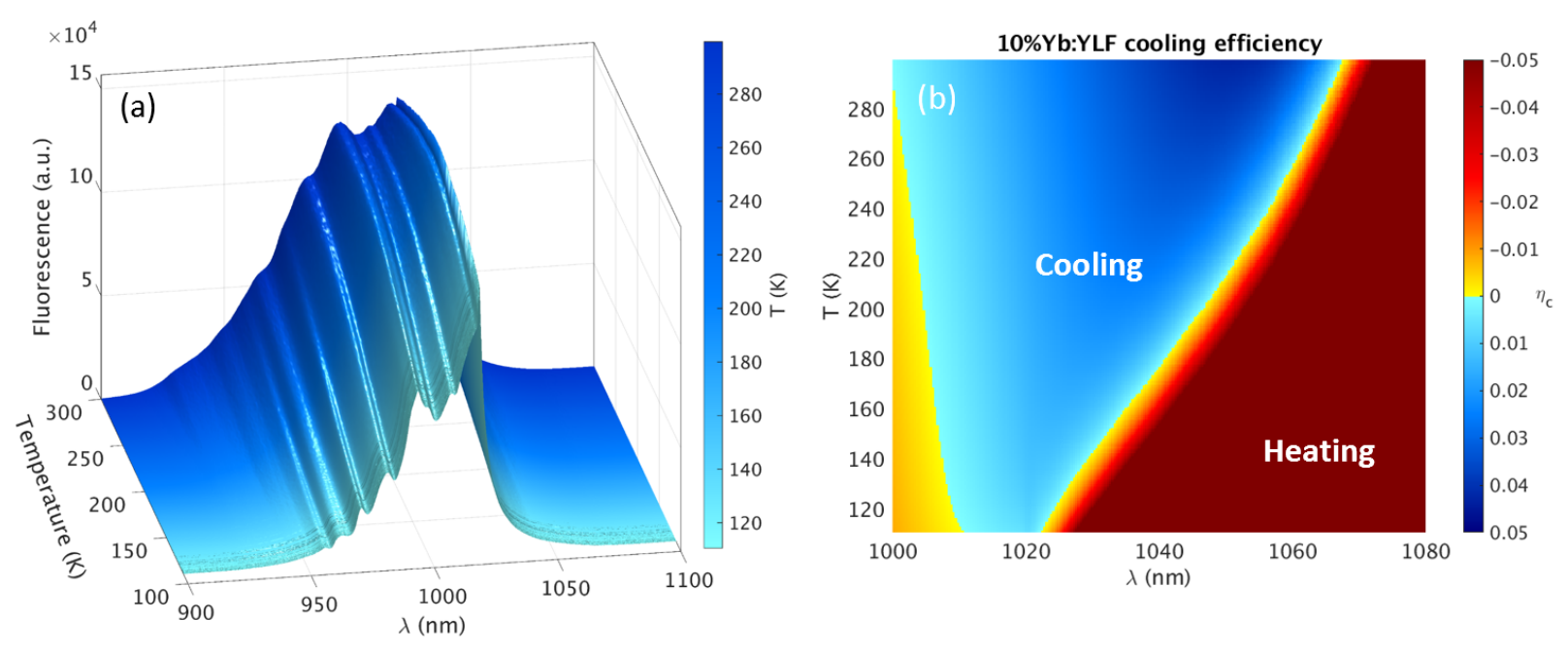

Figure 3. (a) Fluorescence spectra versus temperature. The time interval between each spectra is 5 seconds. (b) Cooling efficiency map $\eta_{c}(\lambda, T)$ for a $10 \%$ wt. $\mathrm{Yb}^{3+}:$ YLF crystal with $\eta_{e x t}=99.6 \%$ and $\alpha_{b}=2 \times 10^{-4} \mathrm{~cm}^{-1} \cdot \eta_{c}(\lambda, T)$ is obtained from fluorescence spectra using McCumber relation ${ }^{30}$ to retrieve $\alpha(\lambda, T)$. Blue regions denote cooling $\left(\eta_{c}>0\right)$, while red regions correspond to heating $\left(\eta_{c}<0\right)$. The global minimum achievable temperature (gMAT) corresponding to $\eta_{c}=0$ is not reached since measurements were taken in room temperature vacuum chamber producing non negligible radiative load. A gMAT of $\sim 93 \mathrm{~K}$ is expected for this crystal. ${ }^{31}$ 


\section{EXPERIMENTAL RESULTS}

\subsection{Experimental set-up}

The laser cooling set-up is outlined in Fig. 4(a). A fiber laser (IPG Photonics, Eckbolsheim, France) generates high energy photons $(20 \mathrm{~W})$ in resonance with the $\mathrm{E} 4 \rightarrow \mathrm{E} 5$ transition $(1020 \mathrm{~nm})$ with a beam diameter of $1 \mathrm{~mm}$ at $1 / e^{2}$. An optical isolator (OI) protects the laser from reflections. A quarter-wave plate (QWP) is used to slightly correct the beam ellipticity. A half-wave plate (HWP), combined with a polarizing beam splitter (PBS), allows to control the polarization axis orientation and the excitation power. The laser beam is then send into a vacuum chamber where a $10 \%$ wt. $\mathrm{Yb}^{3+}: \mathrm{YLF}$ crystal is placed on two bare optical fibers to limit thermal conduction losses. Pressure in the vacuum chamber is lowered to $10^{-5}$ mbar to reduce the convection losses. The beam realizes a round-trip in the crystal, and the transmitted light is collected in a thermal power sensor. We then deduce the absorbed power $P_{a b s}=P_{0}[1-\exp (-2 \alpha L)]$ which depends on the incident power $P_{0}$, the absorption coefficient $\alpha=\alpha_{r}+\alpha_{b}$ and the crystal length $L$. A CMOS camera is used to finely align the laser inside the crystal. Two resistance temperature detectors (RTD) measure the temperature of the crystal holder and the chamber walls in order to calibrate the thermal imaging camera (TC). The fluorescence intensity $I(\lambda, T)$, as shown on Fig. 3(a), is recorded by a fiber spectrometer (Ocean Optics, Lannion, France). An optional polarizer can be placed in front of the fiber to record polarized emission spectra and retrieve $\alpha(\lambda, T)$ for $\mathrm{E} \perp \mathrm{c}$ and E ||$c$ polarizations using McCumber relation. ${ }^{30}$ Finally, a LWIR thermal camera (FLIR, Pontchâteau, France) records the luminance of the crystal, and of a black tape $\left(\epsilon_{b}=0.95\right)$ glued on the holder, trough a germanium window. The temperature range of the thermal camera is $213-423 \mathrm{~K}$, and the noise equivalent temperature difference (NETD) is $<30 \mathrm{mK}$.
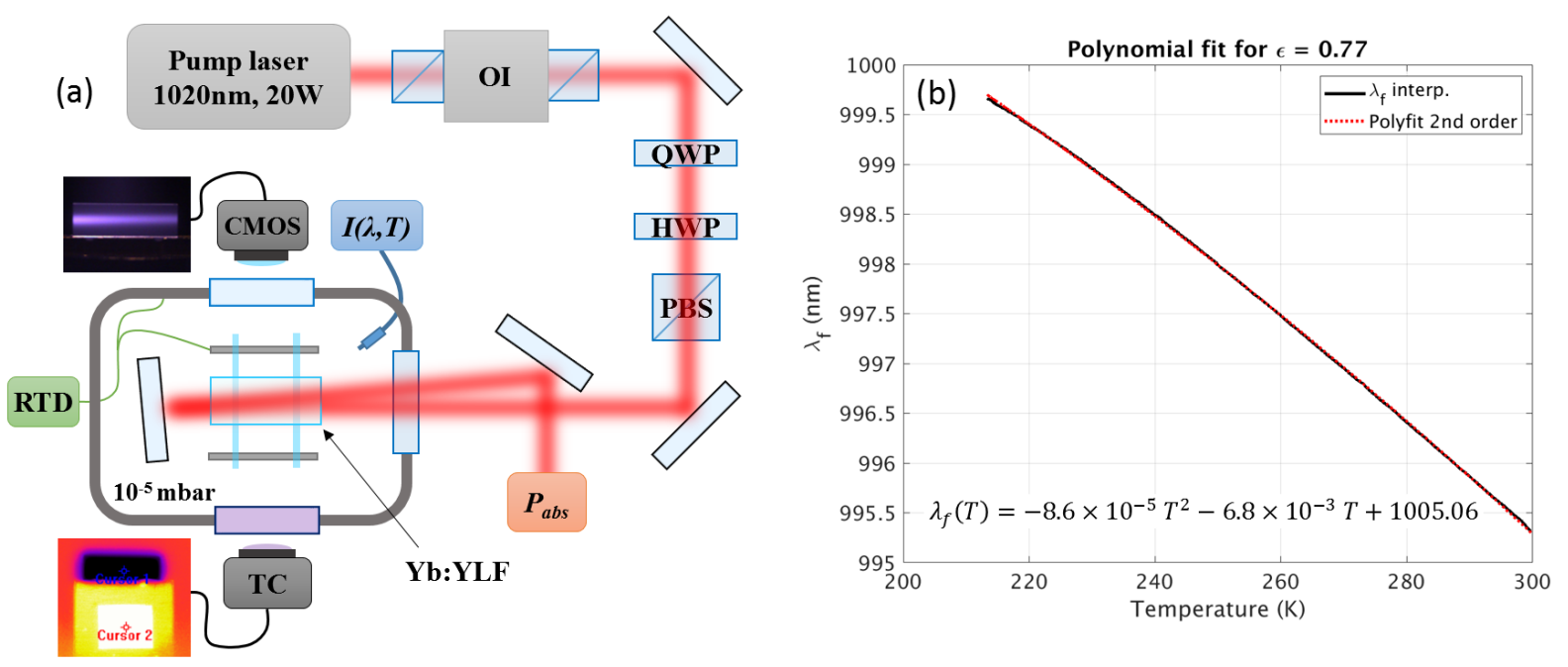

Figure 4. (a) Schematic of the experimental set-up. (b) Temperature dependence of the mean fluorescence for a crystal emissivity of $\epsilon=0.77$. Black line corresponds to an interpolation curve of $\lambda_{f}(T)$ extracted from Fig. 3(a). Red dotted line corresponds to a second order polynomial fit of $\lambda_{f}(T)$.

\subsection{Laser cooling in vacuum}

Fig. 5 shows a recording of crystal and holder temperatures (solid lines) during a laser cooling experiment under vacuum conditions. The saturation of the LWIR camera is clearly visible at $213 \mathrm{~K}$. Temperatures below the saturation is retrieved by measuring the mean fluorescence $\lambda_{f}(T)$ shift in temperature as shown in Fig. 4(b). A second order polynomial fit (red dotted curve) of $\lambda_{f}(T)$ is realized in the $300-213 \mathrm{~K}$ range, in order to extrapolate the temperature below the saturation as illustrated by the blue dotted line in Fig. 5 .

The crystal emissivity is carefully determined by fitting the the warming dynamic of the crystal when the laser is turned off. We assume that black-body radiation is the dominant heat load (Stefan-Boltzmann law), so 


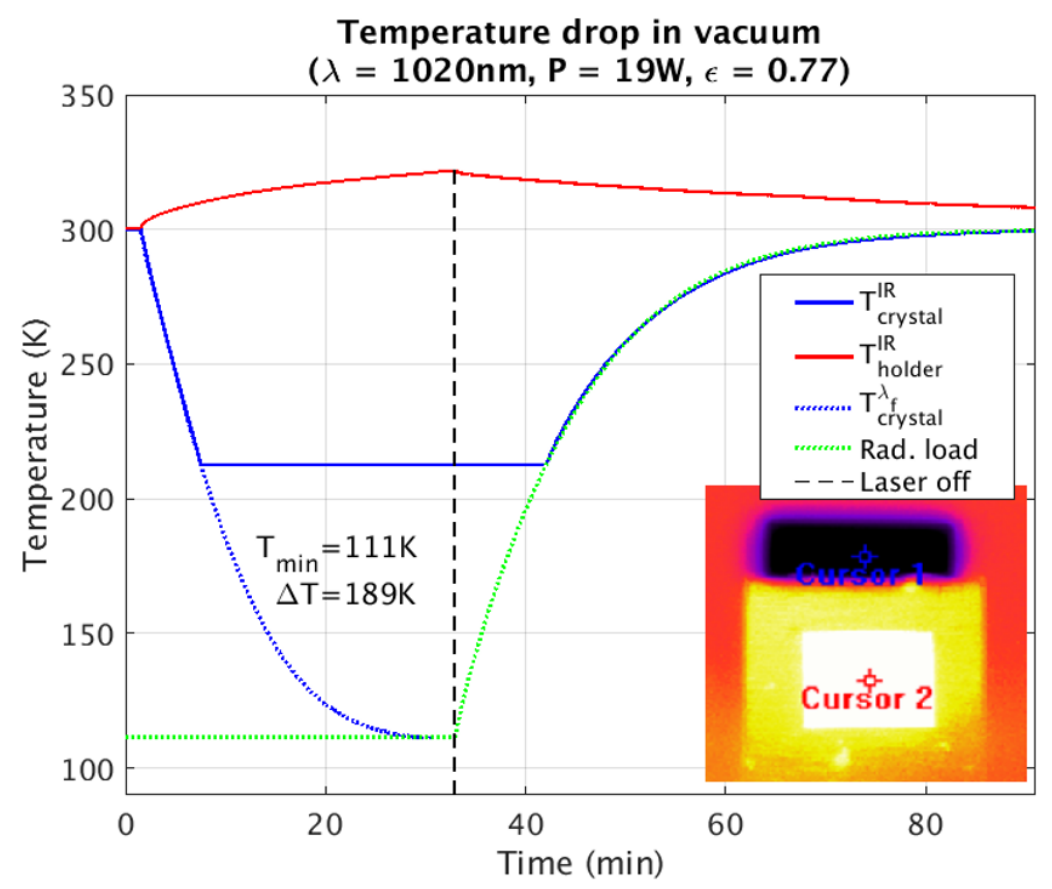

Figure 5. Temperature dynamics under vacuum conditions of a $10 \%$ wt. $\mathrm{Yb}^{3+}$ :YLF crystal (blue) recorded with a thermal imaging camera (solid line) and a spectrometer (dotted line), and for the holder (red). Numerical fit of the crystal warmup is represented by the green dotted line for a crystal emissivity of $\epsilon=0.77$. Inset shows the cursors positions during measurements.

temperature change as

$$
C(T) \frac{d T}{d t}=\frac{\sigma \epsilon A}{1+\chi}\left(T_{c}^{4}-T^{4}\right),
$$

where $C(T)=\rho c_{v}(T) V$ is the heat capacity for a given density $\rho$, specific heat $c_{v}(T)$ and sample volume $V$. $\sigma$ is the Stefan-Boltzmann constant and $T_{c}$ is the environment (chamber) temperature. $\chi=\left(1-\epsilon_{c}\right) \epsilon A / \epsilon_{c} A_{c}$ is a parameter which depends on the surface areas $\left\{A, A_{c}\right\}$ and thermal emissivities $\left\{\epsilon, \epsilon_{c}\right\}$ of the crystal and the chamber respectively. In our case $\chi \approx 0$. Note that radiative load can be reduce by increasing $\chi$ using a tight fitting clamshell with low emissivity walls, ${ }^{31}$ but this kind of technique is out of the scope of our work. A numeric fit (green dotted line) of Eq. 6 leads to an emissivity of $\epsilon=0.77$, comparable with values found in the literature. ${ }^{32}$ Based on this value of the emissivity, a minimum temperature $T_{\min }=111 \mathrm{~K}(\Delta T=189 \mathrm{~K})$ is determined.

\subsection{Laser cooling under atmospheric pressure}

Fig. 6 shows the temperature drop of the same $10 \%$ wt. Yb ${ }^{3+}$ :YLF crystal as shown on Fig. 5 but under atmospheric pressure. To avoid water condensation on the crystal, ambiant air has been replaced by a dry nitrogen atmosphere. In this conditions, the natural convection on the crystal faces limits the temperature drop to $\Delta T \approx 40 \mathrm{~K}$. Assuming that the conduction losses towards fibers are negligible, Eq. 6 is rewritten as follow

$$
C(T) \frac{d T}{d t}=\frac{\sigma \epsilon A}{1+\chi}\left(T_{c}^{4}-T^{4}\right)+A \kappa_{h}\left(T_{c}-T\right)
$$

where $\kappa_{h}$ is the convective heat transfer coefficient. Natural convection simulations, based on the resolution of the coupled Navier-Stokes and heat transfer equations by the finite element method with the commercial software COMSOL (COMSOL AB, Stockholm, Sweden) were performed to determine the value of this coefficient. Good agreement with the experiments is found (black dashed line). Note that the numeric fit of the warming dynamic 
(green dotted line) including the convective load perfectly matches the experimental data and confirm the crystal emissivity.

Let us stress that reaching a temperature drop of $40 \mathrm{~K}$ under atmospheric pressure represents a significant step towards biomedical application since the restrictive vacuum constraint is removed.

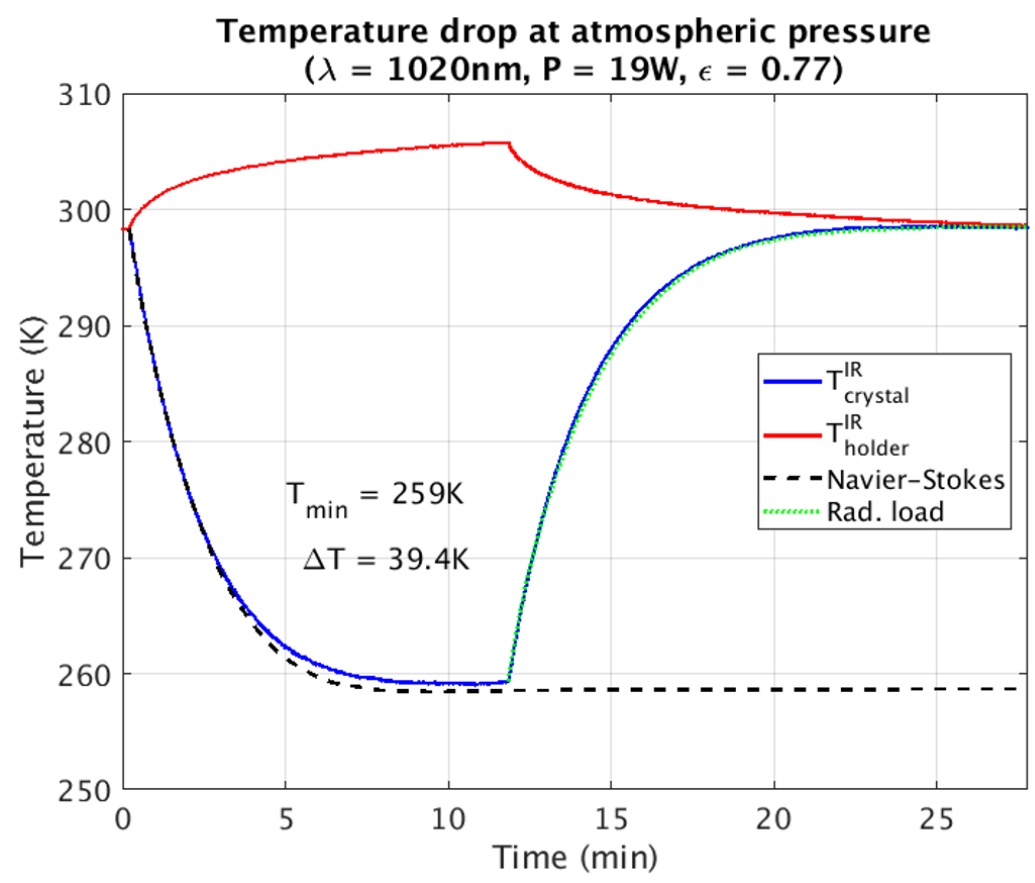

Figure 6. Temperature dynamics of a $10 \%$ wt. $\mathrm{Yb}^{3+}:$ YLF crystal (blue) and the holder (red) under atmospheric pressure. Black dashed line represents the temperature dynamic from finite element resolution of Navier-Stokes and heat transfer equations. Numerical fit of the crystal warm-up is represented by the green dotted line for a crystal emissivity of $\epsilon=0.77$.

\subsection{Laser cooling in biological media}

Laser cooling in a liquid media is very challenging due to significant fluorescence absorption. Since recently, no reference was found in the literature on this subject. In 2015, a group demonstrated for the first time the local refrigeration up to $\Delta T>10 \mathrm{~K}$ of physiological media using hydrothermal $\mathrm{Yb}^{3+}$ :YLF nanocrystals. ${ }^{10}$ Nanocrystals were trapped with optical tweezer and their temperatures were deduced from the analysis of the cold Brownian motion. This demonstration paved the way towards biomedical applications, but biocompatibility issues must be considered first. Since rare-earth doped crystals are mostly toxic and not biocompatible, a careful encapsulation responding to AIMD standards has to be implemented. An other approach might be to change the cooling material. In this way, Kern et al. ${ }^{13}$ demonstrated the potential of nitrogen vacancy doped diamond microcrystals for optical cryocooling. They predicted that cooling of such micro-diamonds could exceed $\Delta T>60 \mathrm{~K}$ for irradiation powers of $300 \mathrm{~mW}$ under vacuum condition. Furthermore, nanodiamonds provide the highest biocompatibility of all carbon-based nanomaterials and are easily functionalizable,${ }^{33,34}$ demonstrating a huge potential for medical diagnostics and treatment. ${ }^{35,36}$

Our strategy is different, as we plan to reduce the size of a $10 \% \mathrm{wt} . \mathrm{Yb}^{3+}: \mathrm{YLF}$ crystal, with the aim of integrating it in a DBS like probe, as shown previously on Fig. 1. A simulation study has been carried out in order to assess the cooling power of such a small crystal. Let us first express the maximum cooling power density $P_{\max }{ }^{37}$

$$
P_{\text {max }} \approx \frac{k_{B} T}{2 \tau_{r}} \frac{N_{t}}{1+e^{\delta E_{g} / k_{B} T}}
$$


(a)
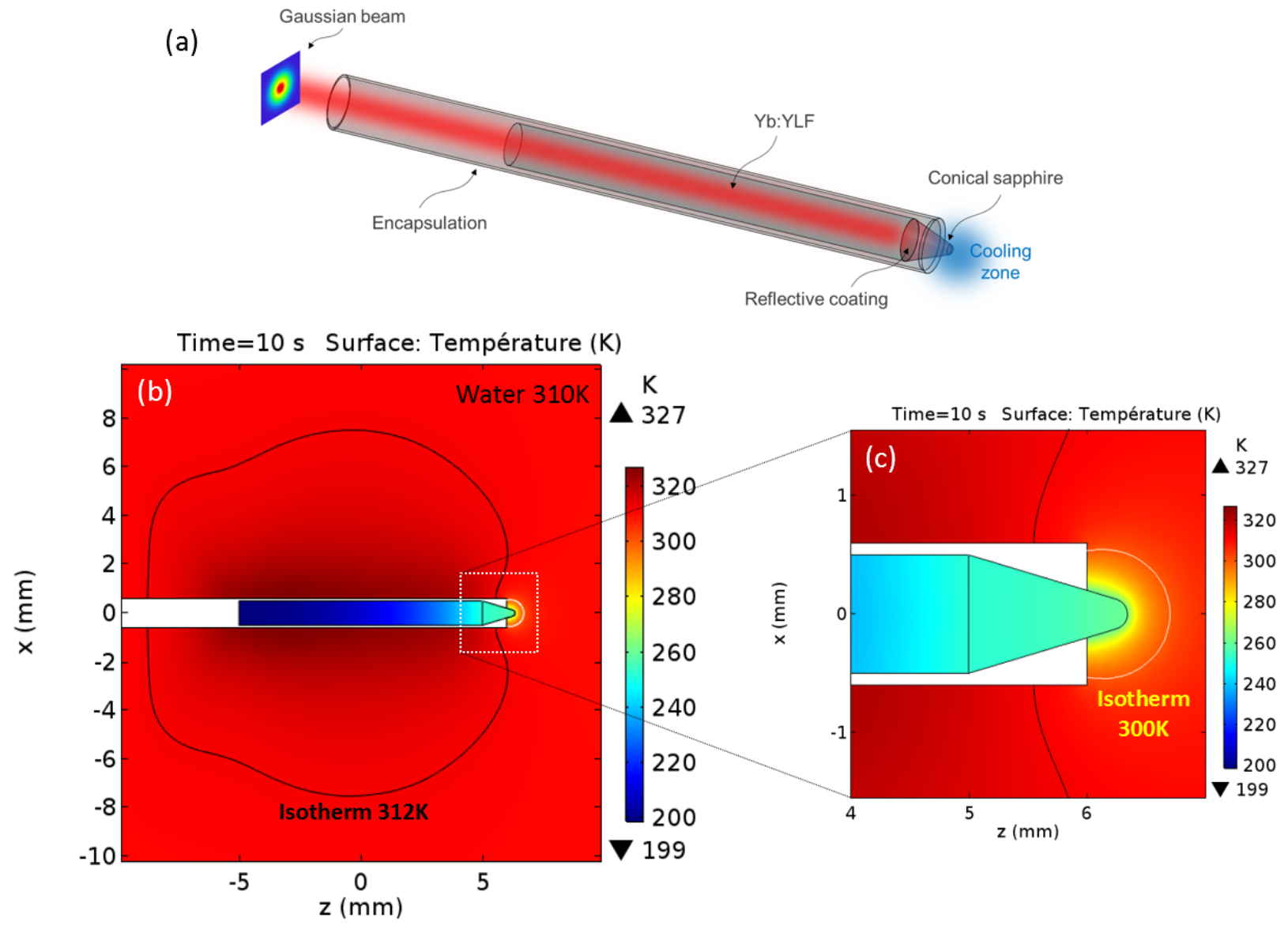

Figure 7. (a) Schematic view of the DBS like cooling probe. (b) Finite element simulations of the DBS like cooling probe after $10 \mathrm{~s}$ of illumination at $19 \mathrm{~W}(1020 \mathrm{~nm})$. Isotherm $312 \mathrm{~K}$ is drawn with a black line. (c) Zoom of the cooling zone below $300 \mathrm{~K}$ delimited by the yellow line.

where $\tau_{r} \approx 3 \mathrm{~ms}$ is the decay rate of $\mathrm{E} 4 \rightarrow \mathrm{E} 5$ transition and $N_{t}$ is the total density population. For a concentration of $10 \%$ wt. of $\mathrm{Yb}^{3+}$ ions, the maximum cooling power density is $\sim 200 \mathrm{~W} . \mathrm{cm}^{-3}$ at $300 \mathrm{~K}$ and $\sim 2 \mathrm{~W} . \mathrm{cm}^{-3}$ at $100 \mathrm{~K}$. Thus, for $\mathrm{a} \sim \mathrm{mm}^{3}$ crystal volume, a maximum cooling power of $\sim 200 \mathrm{~mW}$ can be obtained at room temperature.

Fig. 7 shows an opto-thermal simulation of a realistic cooling probe in $310 \mathrm{~K}$ water representative of the human brain environment. Here, the fluorescence is generated by a Monte Carlo ray tracing with the commercial software OpticStudio (Zemax Europe, Stansted, United Kingdom). The fluorescence is absorbed into a volume detector with an absorption coefficient of $\mu_{a}=0.05 \mathrm{~mm}^{-1}$ corresponding to the near-infrared absorption coefficient of liquid water. ${ }^{38}$ The energy deposited in the detector is then converted into a heat source for finite element simulations.

Here the crystal has a $1 \mathrm{~mm}$ diameter for a length of $10 \mathrm{~mm}$. A vacuum isolation is created by an encapsulation around the crystal. We neglect the fluorescence absorption of the encapsulation. A conical cold finger in sapphire ensure the heat transfer from the water to the cooling crystal. Sapphire is an attractive thermal-link due to its biocompatibility, low optical absorption and high thermal conductivity $\left(\kappa \approx 30 \mathrm{~W} / \mathrm{m} \mathrm{K}\right.$ at $\left.300 \mathrm{~K}^{39}\right)$.

After $10 \mathrm{~s}$, with an input power of $19 \mathrm{~W}$ at $1020 \mathrm{~nm}$, a small volume of $\sim 0.5 \mathrm{~mm}^{3}$ below $\Delta T>10 \mathrm{~K}$ is reached, as illustrated in Fig. 8(a). The cooling volume increases until $30 \mathrm{~s}$ to $\sim 1 \mathrm{~mm}^{3}$ before decreasing since the fluorescence heating takes over. The temperature limit of $312 \mathrm{~K}$ is rapidly reached $(\sim 1 \mathrm{~s})$ and largely exceeded after $30 \mathrm{~s}$, as shown in Fig. 8(b). 

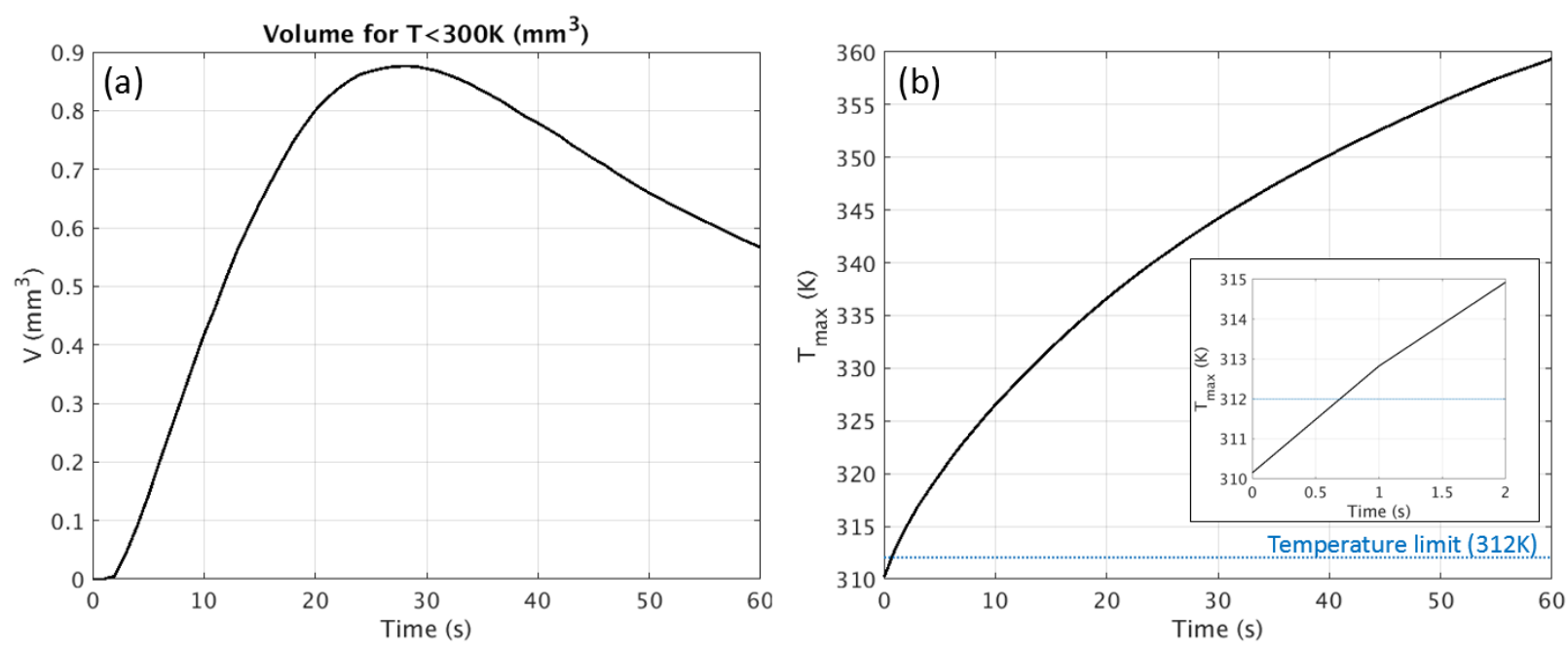

Figure 8. (a) Evolution of the volume of water cooled below $300 \mathrm{~K}$. (b) Evolution of the maximum water temperature during the cooling. Blue dotted line delimits the maximum allowable temperature from ISO14708-1 standard. Inset shows the temperature elevation during the first two seconds. These plots were extracted from finite element simulations presented in Fig. 7.

This simulation study demonstrates the ability of this technology to cool thermal load composed of liquids, but also points out the harmful heating due to fluorescence absorption. Before pushing this technology towards clinical tests, it is necessary to manage this drawback.

\subsection{Fluorescence heating management}

In order to reduce the amount of fluorescence, a new set-up presented in Fig. 9, has been developed. In brief, the temperature of different liquids, close to human brain properties, is regulated at $310 \mathrm{~K}$ with a thermoelectric cooler (TEC). One side wall of the tank containing the liquids is made of germanium in order to ease thermal measurements. On the top of the tank, we either place a silica glass or a high quality dichroic filter (Semrock, Vanves, France). The dichroic filter reflects only the wavelength higher than $\lambda_{f}$, which correspond to $\sim 50 \%$ of the fluorescence (See Fig. 9(c)). Reflected photons are partially reabsorbed by the crystal, and generate an additional cooling since only the anti-Stokes process is possible. The crystal is placed on pieces of optical fiber to reduce the thermal conduction losses.

Blue curves in Fig. 10(a) illustrate this effect by showing a $10 \mathrm{~K}$ improvement using the dichroic filter. Besides of that, we also reduce by a factor two the amount of fluorescence absorbed by the liquid and thus drastically reduce the warming. A temperature reduction of more than $15 \mathrm{~K}$ is measured in water. Note that the temperature limit of $312 \mathrm{~K}$ is only reached after 2 minutes with the filter, whereas it is instantaneously exceeded without it.

To go further, we also measure the fluorescence absorption in several phantoms with various absorption coefficients $\mu_{a}$ and reduced scattering coefficients $\mu_{s}^{\prime}=\mu_{s}(1-g) . \quad \mu_{s}$ is the scattering coefficient and $g$ is the anisotropy factor of the media. Two sets of two calibrated white matter (WM) and gray matter (GM) phantoms based on in vivo, ${ }^{40}$ in vitro ${ }^{41,42}$ and Monte Carlo simulations ${ }^{43}$ from the literature were realized. We choose $\mu_{a}=0.06 \mathrm{~mm}^{-1}$ for GM phantoms and $\mu_{a}=0.09 \mathrm{~mm}^{-1}$ for WM phantoms. Concerning the reduced scattering coefficient, we choose $\mu_{s}^{\prime}=0.6 \mathrm{~mm}^{-1}$ and $\mu_{s}^{\prime}=0.8 \mathrm{~mm}^{-1}$ for GM phantoms and $\mu_{s}^{\prime}=0.8 \mathrm{~mm}^{-1}$ and $\mu_{s}^{\prime}=3.2 \mathrm{~mm}^{-1}$ for WM phantoms. These phantoms were realised with chinese ink, to adjust $\mu_{a}$, and intralipid, to adjust $\mu_{s}^{\prime}$, dissolved in water. The anisotropy factor of the intralipid used is comparable to UHT milk with $g \approx 0.8,{ }^{43}$ which is close to brain anisotropy.

Fig. 10(b) shows the experimental results of the fluorescence absorption in phantoms using the dichroic filter. A comparison is made with the water case (light blue curve) where scattering is almost zero. As expected, no 


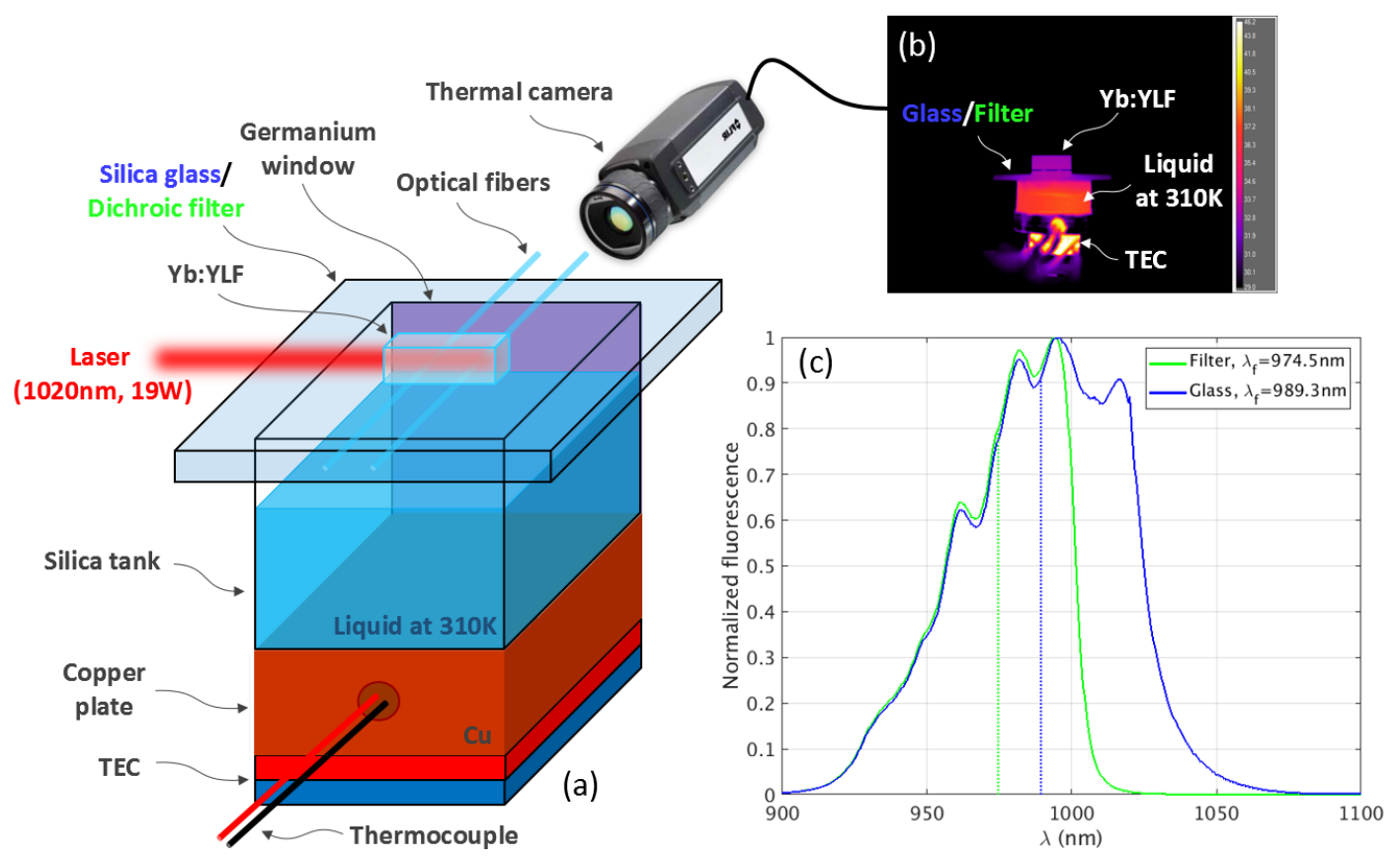

Figure 9. (a) Schematic of the experimental set-up. (b) Thermal image of the experimental set-up during a temperature measurement. (c) Comparison of the fluorescence spectra through a silica glass (blue curve) and a dichroic filter (green curve). The shift of the mean fluorescence corresponds to an enhancement of $\sim 1.5$ of the cooling efficiency.
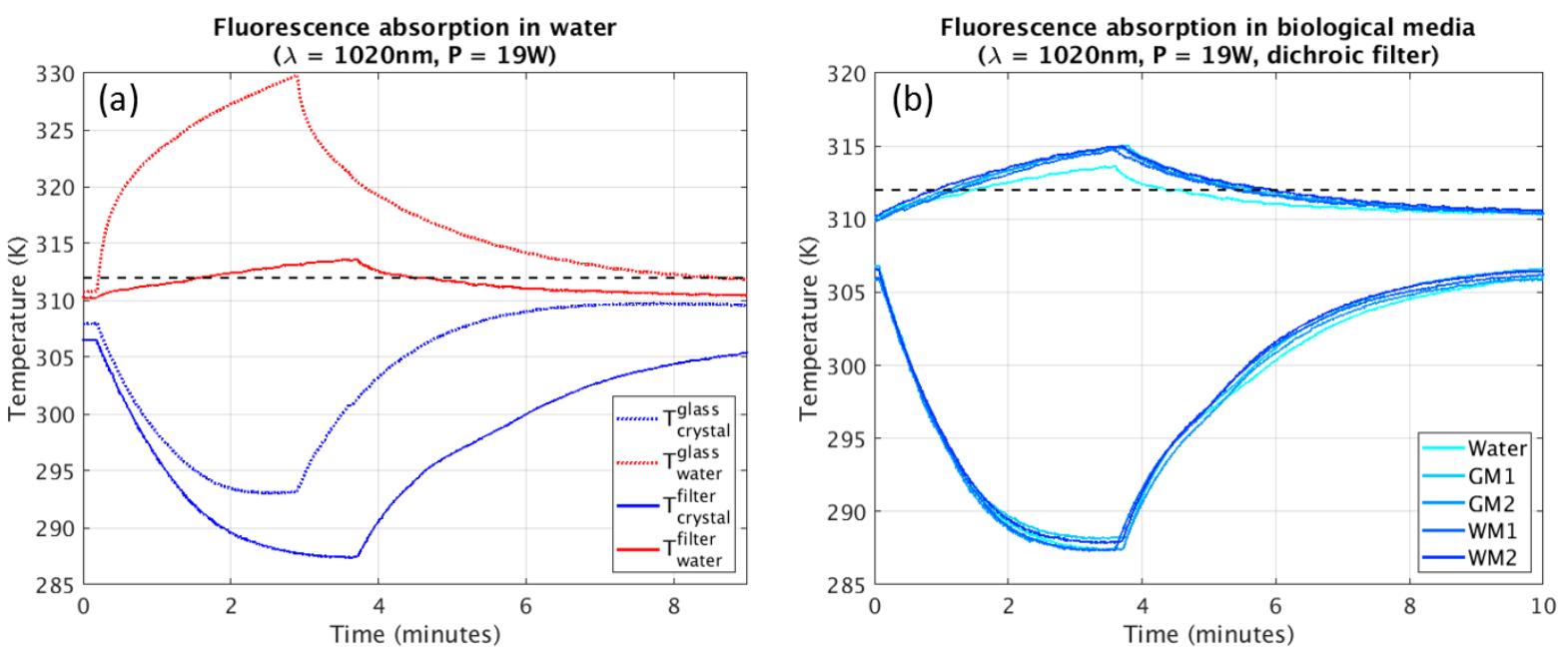

Figure 10. (a) Temperature dynamics of the crystal (blue curves) and water (red curves) when using silica glass (dotted line) and dichroic filter (solid line). (b) Fluorescence heating of various liquids with the dichroic filter. Black dashed line marks the temperature limit of $312 \mathrm{~K}$. Each experiment were realised under an illumination of $19 \mathrm{~W}$ at $1020 \mathrm{~nm}$.

difference can be seen on the cooling dynamics of the crystal. Concerning the fluorescence heating, no significant differences appear between the phantoms. However, we notice that water heats slower than phantoms. This is mostly due to scattering since the water absorption coefficient $\mu_{a} \approx 0.05 \mathrm{~mm}^{-1}$ is very close to that of WM phantoms. 


\section{CONCLUSION}

In this paper, we report the first possible biomedical application of optical refrigeration. We demonstrate the possibility to cool a volume of a $\mathrm{mm}^{3}$ below $300 \mathrm{~K}$ in a deep brain region inaccessible so far by other cooling technologies. This result is an important achievement towards the realization of a fully implantable device able to treat medically refractory epilepsy. In addition, we propose a solution based on dichroic filtering to drastically reduce the harmful heating induced by the fluorescence absorption and to enhance by $70 \%$ the cooling efficiency. Beside of that, these results pave the way towards new medical approaches using optical cooling for cryosurgery or cryotherapy in deep brain region.

\section{ACKNOWLEDGMENTS}

This work was supported by the French CEA program Carnot Explorer and the endowment fund of Clinatec (CEA Grenoble, France). The authors thank D. Rytz from FEE (Idar-Oberstein, Germany) for supplying the best quality crystals available. The authors would also like to acknowledge C. Emain from CEA-LETI for her helpful contribution in the realization and characterisation of the brain phantoms.

\section{REFERENCES}

[1] Epstein, R. I., Buchwald, M. I., Edwards, B. C., Gosnell, T. R., and Mungan, C. E., "Observation of laser-induced fluorescent cooling of a solid," Nature 377, 500-503 (Oct. 1995).

[2] Mungan, C. E., Buchwald, M. I., Edwards, B. C., Epstein, R. I., and Gosnell, T. R., "Laser Cooling of a Solid by 16 K Starting from Room Temperature," Physical Review Letters 78, 1030-1033 (Feb. 1997).

[3] Hoyt, C. W., Sheik-Bahae, M., Epstein, R. I., Edwards, B. C., and Anderson, J. E., "Observation of Anti-Stokes Fluorescence Cooling in Thulium-Doped Glass," Physical Review Letters 85, 3600-3603 (Oct. 2000).

[4] Thiede, J., Distel, J., Greenfield, S. R., and Epstein, R. I., "Cooling to 208k by optical refrigeration," Applied Physics Letters 86, 154107 (Apr. 2005).

[5] Fernandez, J., Garcia-Adeva, A. J., and Balda, R., "Anti-Stokes Laser Cooling in Bulk Erbium-Doped Materials," Physical Review Letters 97, 033001 (July 2006).

[6] Filho, E. S. d. L., Krishnaiah, K. V., Ledemi, Y., Yu, Y.-J., Messaddeq, Y., Nemova, G., and Kashyap, R., "Ytterbium-doped glass-ceramics for optical refrigeration," Optics Express 23, 4630 (Feb. 2015).

[7] Patterson, W., Bigotta, S., Sheik-Bahae, M., Parisi, D., Tonelli, M., and Epstein, R., "Anti-Stokes luminescence cooling of $\mathrm{Tm}^{3+}$ doped $\mathrm{BaY}_{2} \mathrm{~F}_{8}, "$ Optics Express 16, 1704-1710 (Feb. 2008).

[8] Condon, N. J., Bowman, S. R., O'Connor, S. P., Quimby, R. S., and Mungan, C. E., "Optical cooling in $\mathrm{Er}^{3+}: \mathrm{KPb}_{2} \mathrm{Cl}_{5}, "$ Optics Express 17, 5466-5472 (Mar. 2009).

[9] Seletskiy, D. V., Melgaard, S. D., Bigotta, S., Di Lieto, A., Tonelli, M., and Sheik-Bahae, M., "Laser cooling of solids to cryogenic temperatures," Nature Photonics 4, 161-164 (Mar. 2010).

[10] Roder, P. B., Smith, B. E., Zhou, X., Crane, M. J., and Pauzauskie, P. J., "Laser refrigeration of hydrothermal nanocrystals in physiological media," Proceedings of the National Academy of Sciences of the United States of America 112, 15024-15029 (Dec. 2015).

[11] Ha, S.-T., Shen, C., Zhang, J., and Xiong, Q., "Laser cooling of organic-inorganic lead halide perovskites," Nature Photonics 10, 115-121 (Feb. 2016).

[12] Melgaard, S. D., Albrecht, A. R., Hehlen, M. P., and Sheik-Bahae, M., "Solid-state optical refrigeration to sub-100 Kelvin regime," Scientific Reports 6, 20380 (Feb. 2016).

[13] Kern, M., Jeske, J., Lau, D. W. M., Greentree, A. D., Jelezko, F., and Twamley, J., "Optical cryocooling of diamond," Physical Review B 95, 235306 (June 2017).

[14] Zhang, J., Li, D., Chen, R., and Xiong, Q., "Laser cooling of a semiconductor by 40 kelvin: an optical refrigerator based on cadmium sulfide nanoribbions," 863808 (Mar. 2013).

[15] Filho, E. S. d. L., Nemova, G., Loranger, S., and Kashyap, R., "Laser-induced cooling of a Yb:YAG crystal in air at atmospheric pressure," Optics Express 21, 24711-24720 (Oct. 2013).

[16] Zhong, B., Luo, H., Shi, Y., and Yin, J., "Laser cooling of 5 mol. \% Yb ${ }^{3+}$ : LuLiF 4 crystal in air," Optical Engineering 56, 011102 (Aug. 2016). 
[17] Hehlen, M. P., Meng, J., Albrecht, A. R., Lee, E. R., Gragossian, A., Love, S. P., Hamilton, C. E., Epstein, R. I., and Sheik-Bahae, M., "First demonstration of an all-solid-state optical cryocooler," Light: Science \& Applications 7, 15 (June 2018).

[18] Rothman, S. M., Smyth, M. D., Yang, X.-F., and Peterson, G. P., "Focal cooling for epilepsy: An alternative therapy that might actually work," Epilepsy 83 Behavior 7, 214-221 (Sept. 2005).

[19] Smyth, M. D., Han, R. H., Yarbrough, C. K., Patterson, E. E., Yang, X.-F., Miller, J. W., Rothman, S. M., and D'Ambrosio, R., "Temperatures Achieved in Human and Canine Neocortex During Intraoperative Passive or Active Focal Cooling," Therapeutic Hypothermia and Temperature Management 5, 95-103 (June 2015).

[20] Bell, G. and Sander, J., "CPD - Education and self-assessment The epidemiology of epilepsy: the size of the problem," Seizure 10, 306-316 (June 2001).

[21] WHO, "Epilepsy. https://www.who.int/news-room/fact-sheets/detail/epilepsy."

[22] Cockerell, O. C., Sander, J. W. A. S., Hart, Y. M., Shorvon, S. D., and Johnson, A. L., "Remission of epilepsy: results from the National General Practice Study of Epilepsy," The Lancet 346, 140-144 (July 1995).

[23] Kwan, P. and Sperling, M. R., "Refractory seizures: Try additional antiepileptic drugs (after two have failed) or go directly to early surgery evaluation?," Epilepsia 50(s8), 57-62 (2009).

[24] Engel, J., "Why Is There Still Doubt to Cut It Out?," Epilepsy Currents 13(5), 198-204 (2013).

[25] Amar, A. P., Apuzzo, M. L. J., and Liu, C. Y., "Vagus nerve stimulation therapy after failed cranial surgery for intractable epilepsy: results from the vagus nerve stimulation therapy patient outcome registry," Neurosurgery 55, 1086-1093 (Nov. 2004).

[26] Salanova, V., Witt, T., Worth, R., Henry, T. R., Gross, R. E., Nazzaro, J. M., Labar, D., Sperling, M. R., Sharan, A., Sandok, E., Handforth, A., Stern, J. M., Chung, S., Henderson, J. M., French, J., Baltuch, G., Rosenfeld, W. E., Garcia, P., Barbaro, N. M., Fountain, N. B., Elias, W. J., Goodman, R. R., Pollard, J. R., Tröster, A. I., Irwin, C. P., Lambrecht, K., Graves, N., and Fisher, R., "Long-term efficacy and safety of thalamic stimulation for drug-resistant partial epilepsy," Neurology 84, 1017-1025 (Mar. 2015).

[27] Fujii, M., Fujioka, H., Oku, T., Tanaka, N., Imoto, H., Maruta, Y., Nomura, S., Kajiwara, K., Saito, T., Yamakawa, T., Yamakawa, T., and Suzuki, M., "Application of focal cerebral cooling for the treatment of intractable epilepsy," Neurologia Medico-Chirurgica 50(9), 839-844 (2010).

[28] Pringsheim, P., "Zwei Bemerkungen über den Unterschied von Lumineszenz- und Temperaturstrahlung," Zeitschrift für Physik 57, 739-746 (Nov. 1929).

[29] Sheik-Bahae, M. and Epstein, R. I., "Optical refrigeration," Nature Photonics 1, 693-699 (Dec. 2007).

[30] McCumber, D. E., "Einstein Relations Connecting Broadband Emission and Absorption Spectra," Physical Review 136, A954-A957 (Nov. 1964).

[31] Melgaard, S. D., Cryogenic optical refrigeration: Laser cooling of solids below 123 K, ph.D., The University of New Mexico, United States - New Mexico (2013).

[32] Seletskiy, D. V., Melgaard, S. D., Sheik-Bahae, M., Bigotta, S., Lieto, A. D., and Tonelli, M., "Laser Cooling of a Semiconductor Load to $165 \mathrm{~K}$, " in [Conference on Lasers and Electro-Optics 2010 (2010), paper QFG1], QFG1, Optical Society of America (May 2010).

[33] Vaijayanthimala, V. and Chang, H.-C., "Functionalized fluorescent nanodiamonds for biomedical applications," Nanomedicine (London, England) 4, 47-55 (Jan. 2009).

[34] Zhu, Y., Li, J., Li, W., Zhang, Y., Yang, X., Chen, N., Sun, Y., Zhao, Y., Fan, C., and Huang, Q., "The Biocompatibility of Nanodiamonds and Their Application in Drug Delivery Systems," Theranostics 2(3), 302-312 (2012).

[35] Narayan, R. J., Boehm, R. D., and Sumant, A. V., "Medical applications of diamond particles \& surfaces," Materials Today 14, 154-163 (Apr. 2011).

[36] Tsai, L.-W., Lin, Y.-C., Perevedentseva, E., Lugovtsov, A., Priezzhev, A., and Cheng, C.-L., "Nanodiamonds for Medical Applications: Interaction with Blood in Vitro and in Vivo," International Journal of Molecular Sciences 17 (July 2016).

[37] Seletskiy, D. V., Hehlen, M. P., Epstein, R. I., and Sheik-Bahae, M., "Cryogenic optical refrigeration," Advances in Optics and Photonics 4, 78 (Mar. 2012). 
[38] Curcio, J. A. and Petty, C. C., "The Near Infrared Absorption Spectrum of Liquid Water," Journal of the Optical Society of America 41, 302 (May 1951).

[39] Dobrovinskaya, E. R., Lytvynov, L. A., and Pishchik, V., [Sapphire: Material, Manufacturing, Applications], Micro- and Opto-Electronic Materials, Structures, and Systems, Springer US (2009).

[40] Bevilacqua, F., Piguet, D., Marquet, P., Gross, J. D., Tromberg, B. J., and Depeursinge, C., "In vivo local determination of tissue optical properties: applications to human brain," Applied Optics 38, 4939-4950 (Aug. 1999).

[41] Yaroslavsky, A. N., Schulze, P. C., Yaroslavsky, I. V., Schober, R., Ulrich, F., and Schwarzmaier, H.-J., "Optical properties of selected native and coagulated human brain tissues in vitro in the visible and near infrared spectral range," Physics in Medicine and Biology 47, 2059-2073 (June 2002).

[42] Gebhart, S. C., Lin, W. C., and Mahadevan-Jansen, A., "In vitro determination of normal and neoplastic human brain tissue optical properties using inverse adding-doubling," Physics in Medicine 83 Biology 51(8), 2011 (2006).

[43] Johansson, J. D., "Spectroscopic method for determination of the absorption coefficient in brain tissue," Journal of Biomedical Optics 15, 057005 (Oct. 2010). 